\title{
A new self-made digital slide scanner and microscope for imaging and quantification of fluorescent microspheres
}

\author{
William Henning ${ }^{1}$, Julie Bjerglund Andersen ${ }^{1}$, Liselotte Højgaard ${ }^{1}$, Gorm Greisen ${ }^{2}$, Ian Law ${ }^{1}$, Anders Thorseth ${ }^{3}$, \\ Anders Nymark Christensen*4 \\ ${ }^{1}$ Department of Clinical Physiology, Nuclear Medicine and PET, Rigshospitalet, University of Copenhagen, Denmark \\ ${ }^{2}$ Department of Neonatology, Rigshospitalet, University of Copenhagen, Denmark \\ ${ }^{3}$ Department of Photonics Engineering, Technical University of Denmark, Denmark \\ ${ }^{4}$ Department of Applied Mathematics and Computer Science, Technical University of Denmark, Denmark
}

Received: May 1, 2015

DOI: $10.5430 / j b g c . v 5 n 2 p 33$
Accepted: June 27, 2015

Online Published: September 21, 2015

URL: http://dx.doi.org/10.5430/jbgc.v5n2p33

\begin{abstract}
Objective: A low-cost microscope slide scanner was constructed for the purpose of digital imaging of newborn piglet brain tissue and to quantify fluorescent microspheres in tissue.

Methods: Using a standard digital single-lens reflex (DSLR) camera, fluorescent imaging of newborn piglet brain tissue was performed. A computer algorithm available for download was created to detect fluorescent microspheres in the brain tissue slides and to calculate regional cerebral blood flow ( $\mathrm{rCBF}$ ). The precision of the algorithm was tested by comparing with manual counting of the fluorescent microspheres. Finally, bright-field imaging was tested by adding light diffuser film.

Results: Cost of the slide scanner was a fraction of the cost of a commercial slide scanner. The slide scanner was able to image a large number of tissue slides in a semiautomatic manner and provided a large field of view (FOV) of $101 \mathrm{~mm}^{2}$ combined with a resolution of $2.9 \mu \mathrm{m}$. The mean difference (SD) between manual and automatic counts was in absolute numbers 0.32 (1.5) microspheres ranging from -5 to 5 microspheres per slide. The relative total difference between automatic and manual counts was $-3.1 \%$.

Conclusions: A slide scanner was constructed and an automatic algorithm to detect fluorescent microspheres in tissue was developed and validated and showed an acceptable difference to "gold standard" manual counting. The slide scanner can be regarded as a low-cost alternative for researchers when digital slide imaging and quantification of fluorescent microspheres are needed.
\end{abstract}

Key Words: Fluorescence, Slide scanner, Microspheres, Microscopy, Cerebral blood flow, Bright-field

\section{Introduction}

Digital microscopy has a wide array of applications. The image can be reviewed by others with the ease of digital image sharing and several recent results point to the efficacy of automated analysis of histological samples once they have been digitalized. ${ }^{[1,2]}$ Fluorescence microscopy enables a wide array of staining techniques to test e.g. gene expression, proteins, and RNA sequences.

\footnotetext{
*Correspondence: Anders Nymark Christensen; Email: anym@dtu.dk; Address: Department of Applied Mathematics and Computer Science, Technical University of Denmark, Richard Petersens Plads, bygning 324, 2800 Kgs. Lyngby, Denmark.
} 
This article presents a low-cost slide scanner for digital imaging build for the specific purpose of fluorescent microsphere detection and blood flow estimation, but with a wide range of possible applications in microscopy. The cost was approximately USD 1,000, which is a fraction of the cost of a commercial system.

\section{Method}

\subsection{Motivating study}

We constructed the slide scanner for fluorescent microsphere detection, which is a niche application in medical science for tissue and organ blood flow estimation. ${ }^{[3]}$ In brief, the fluorescent microsphere are $15 \mu \mathrm{m}$ polystyrene particles, which are injected into the left ventricle of an animal and carried through the bloodstream to tissues, where they are trapped in the capillaries and serve as a surrogate measure of tissue blood flow. Ex-vivo the tissue of interest is usually digested and the microspheres counted by flow cytometry. With a reference blood sample drawn simultaneously with the injection, an estimate of the quantitative blood flow to the tissue of interest can be made. ${ }^{[1,4]}$ Alternatively the tissue of interest is sliced and the fluorescent microspheres can be counted in-situ with a slide scanner. The quantification of tissue blood flow can be done by the following calculation:

$$
F=\frac{W_{\text {rate }} N_{\text {tissue }}}{N_{\text {blood }} V_{\text {tissue }}}
$$

where $F$ is tissue blood flow $(\mathrm{ml} / \mathrm{min} / 100 \mathrm{ml}), W_{\text {rate }}$ is the withdrawal rate of the reference blood sampling $(\mathrm{ml} / \mathrm{min})$, $N_{\text {tissue }}$ and is the number of microspheres in the tissue and $N_{\text {blood }}$ is the number of microspheres in the reference blood, and $V_{\text {tissue }}$ is the volume of the tissue $(100 \mathrm{ml})$.

A study determining the regional cerebral blood flow (rCBF) in newborn piglets was carried out. Table 1 shows the specifications for the fluorescent microspheres used in the study. Brain tissue was excised and sliced in a cryotome. The commercial solutions for imaging of slides proved too costly and led to the development of the slide scanner in the present study. As an addition to the slide scanner itself, image processing algorithms were developed to detect and count the fluorescent microspheres in the images. In this manner, estimation of rCBF could be done semiautomatically using the slide scanner and algorithm.

Both carmine/red and yellow/green fluorescent microspheres have a long tail in the excitation spectrum, which made it possible to excite them with light below $400 \mathrm{~nm}$ wavelength. In the slide scanner blue fluorescent microspheres showed the smallest contrast because of the emission value close to $400 \mathrm{~nm}$.
Table 1: The dominant excitation and emission wavelengths for the fluorescent microsphere colors used in our study of rCBF in newborn piglets

\begin{tabular}{lll}
\hline $\begin{array}{l}\text { Fluorescent } \\
\text { microspheres }\end{array}$ & $\begin{array}{l}\text { Excitation } \\
\text { wavelength }\end{array}$ & $\begin{array}{l}\text { Emission } \\
\text { wavelength }\end{array}$ \\
\hline Carmine/Red & $580 \mathrm{~nm}$ & $620 \mathrm{~nm}$ \\
Yellow/Green & $505 \mathrm{~nm}$ & $515 \mathrm{~nm}$ \\
Blue & $365 \mathrm{~nm}$ & $415 \mathrm{~nm}$ \\
\hline
\end{tabular}

\subsection{Set-up}

Materials needed for constructing the slide scanner: a sturdy frame (dimensions approximately $40 \mathrm{~cm} \times 40 \mathrm{~cm} \times 60 \mathrm{~cm}$ ), an adjustable camera mounting, two UV light bulbs (Omnilux, UK), an optical shortpass $400 \mathrm{~nm}$ cutoff glass filter (Edmund Optics, USA), a frosted glass plate, a standard digital single-lens reflex (DSLR) camera and objective lens of 18 mm - 55 mm (EOS 1100D, Canon, Japan), light diffuser film and lens extension tubes (MeiKe, MK-C-AF1-A).

For bright-field images, testing using microscope objective lenses (Zeiss EC Plan-Neofluar $40 \times / 0.75$ and Olympus LCAch P\&P $20 \times / 0.4)$ were carried out with the use of a microscope objective thread adapter, thus enabling direct mounting of microscope objective lenses on the camera objective lens. A USB-cable connected the camera to a computer with acquisition software (Canon, Japan). The set-up was protected from external sources of light with covers. The primary camera settings (Aperture, Shutter speed, and ISO) for optimal fluorescent microsphere detection were tested in two $3^{3}$ factorial designs to optimize visualization of the fluorescent microspheres. See Figure 1 for details of the set-up.

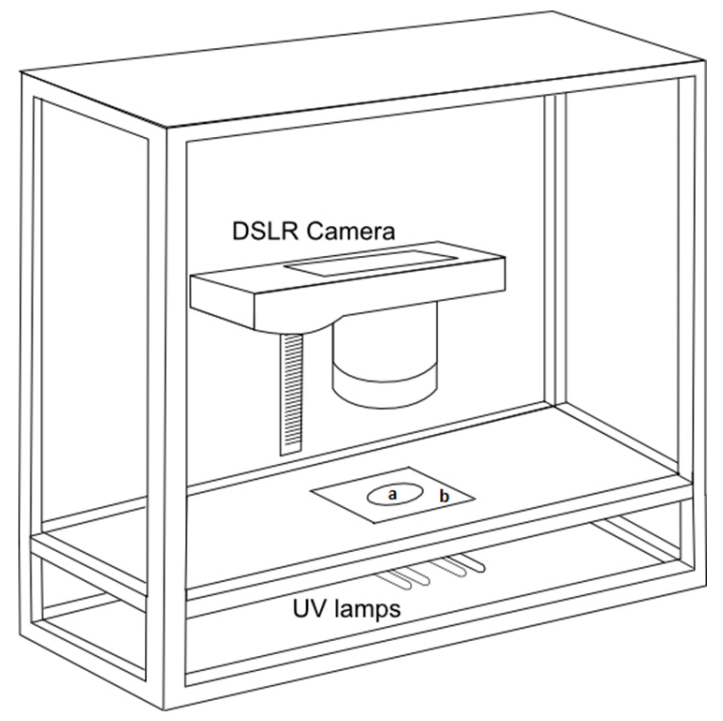

Figure 1: Set-up of the self-made slide scanner A sturdy frame, DSLR-camera mounted on a slide and UV light bulbs along with shortpass cutoff glass filter (a) and frosted glass plate (b). 


\subsection{Algorithm}

Fluorescent microsphere detection algorithms were developed in MATLAB (Mathworks, MA, USA). Initially, tissue borders were automatically delineated for estimation of the tissue area. Detection of fluorescent microspheres relied on several data processing steps using the following parameters: Firstly, a light intensity cutoff value for microspheres was set. Secondly, maximum and minimum pixel areas of microspheres were defined. Thirdly, estimation of microsphere roundness was given by:

$$
\text { Ratio }=\left|\frac{C}{D_{\text {small }}}-\pi\right|
$$

where $D_{\text {small }}$ denotes the lower cutoff diameter of the object in question and $C$ denotes the object circumference. If an object is a perfect circle, the ratio is zero. If an object deviates from a perfect circle, the ratio was higher than zero. The cut-off ratio for roundness of microspheres was set to 2 . These parameters ensured differentiation between all other tissue structures than microspheres, minimizing any falsely detected microspheres. Finally, when an object was classified as a microsphere, an algorithm determining the color of fluorescence was applied. As each image was a RGB layer image (derived from the red, green and blue color model in the images), the light intensity value of each microsphere was used as a parameter for color determination of the fluorescence. Figure 2 shows an example of the RGB values of the three colors. In combination, all parameters ensured an excellent detection performance of fluorescent microspheres, which was validated in a sample series (see Results section).

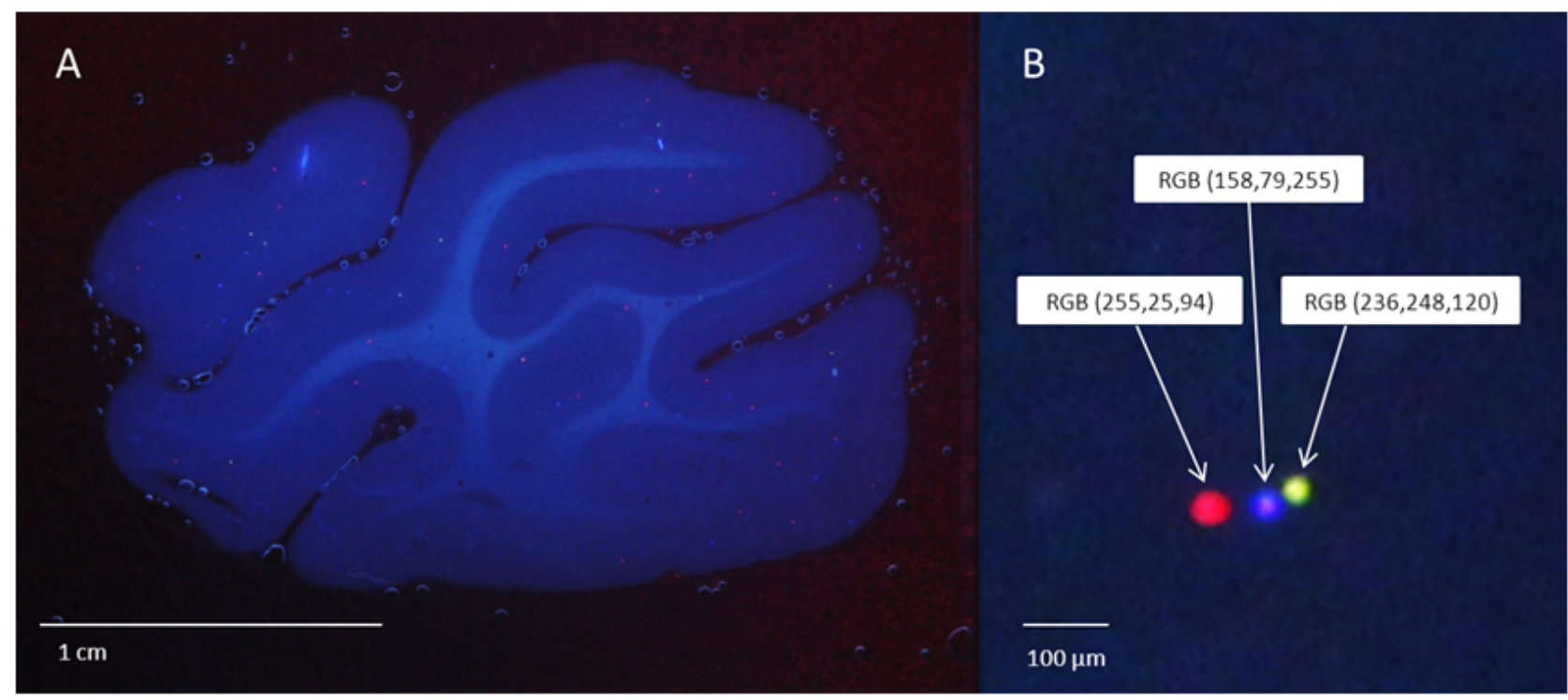

Figure 2: Newborn piglet brain, $200 \mu \mathrm{m}$ slice of a left frontal region

A. No staining was applied in the preparation of the slide. B. Carmine/Red, Blue and Yellow/Green fluorescent microspheres are shown along with corresponding RGB values (derived from the Red, Green and Blue color model in the image), which was used to determine the fluorescent color of the microspheres. Technique: DSLR camera with extensions and UV light bulbs. The resolution was measured at $5.7 \mu \mathrm{m}$. Used for automatic detection of fluorescent microspheres for determination of $r C B F$.

Image analysis of a right or left hemisphere of a piglet brain (approximately 250 slides) in MATLAB was completed in approximately 20 minutes using a powerful computer (Intel I7-2600, 3.4 GHz processor and $16 \mathrm{~GB}$ RAM) and MATLAB's parallel computing toolbox. ${ }^{[5]}$ The algorithm is available for download from MathWorks online library (http://www. mathworks.com/matlabcentral/file exchange/48760-blobdetection-for-bloodflow-e stimation) and also from GitHubs open source code management in Python code (https://github.com/AndersN ymarkChristensen/BlobDetectionForBloodFlow).

\subsection{Specifications}

\section{Spectra}

The spectral power distribution of the UV light source was characterized using a vendor calibrated double monochromator system (SPECTRO 320, Instrument Systems, Germany) with an input optic for spectral irradiance measurements. The wavelength resolution of the monochromator is approximately $1 \mathrm{~nm}$. The results are seen in Figure 3 . Figure 3a shows a distinct peak just above $400 \mathrm{~nm}$ for the unmodified UV light (set-up 1). This peak coincides with the emission wavelength of the blue microspheres and was successfully removed by the shortpass cutoff filter (set-up 
$2)$. Figure $3 b$ shows the light on logarithmic scale. In set-up 3 , the addition of a light diffuser film is shown and a smooth shoulder from $400 \mathrm{~nm}$ with higher irradiance values than set-up 1 and set-up 2 is seen. This gave a bright-field-like lighting when compared with set-up 1 and 2 and enabled bright-field imaging.

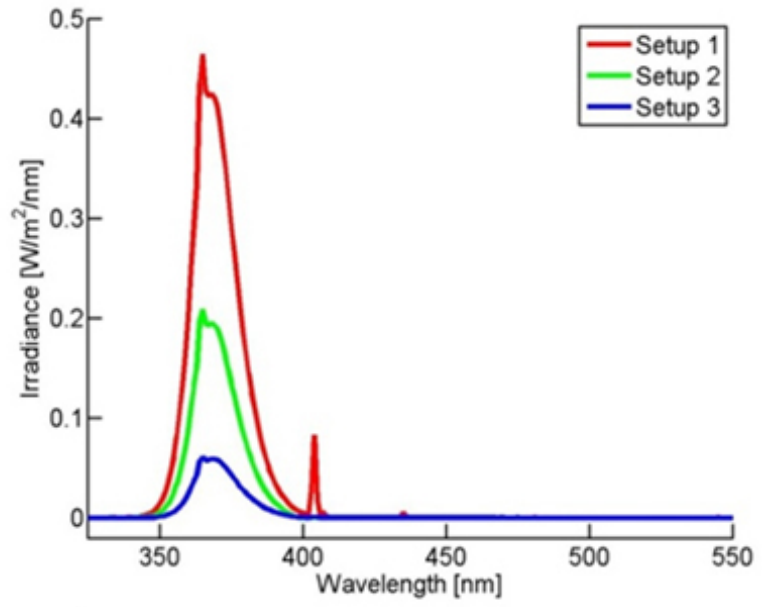

A

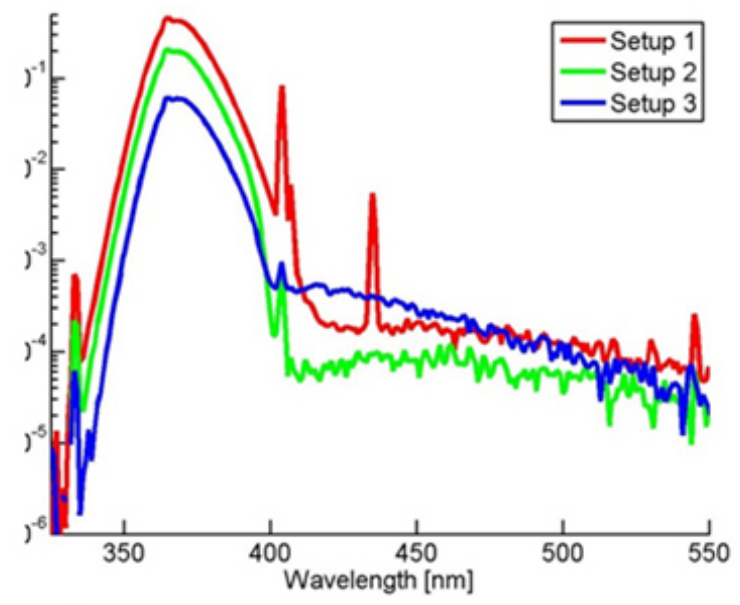

B

Figure 3: The spectral power distribution of the light source (UV light bulbs) measured at a distance of $12.5 \mathrm{~cm}$ A. Linear scale, B. Logarithmic scale. The following set-ups are displayed: Set-up 1. UV light bulbs without modifications (red line), Set-up 2. UV light bulbs with a shortpass $400 \mathrm{~nm}$ cutoff filter (green line), Set-up 3. UV light bulbs with cutoff shortpass filter and light diffuser film (blue line). Note the higher irradiance value of set-up 3 (blue line) at $400 \mathrm{~nm}$ as seen in b, which enabled bright-field imaging.

\section{Results}

\subsection{Resolution}

Magnification depended on the extension tubes used with the camera. The more - and there by longer - extension tubes used, the greater the magnification and hence image resolution. However, the camera-sensor also affects the final image resolution. We have measured the image resolution in pixel width. The image sensor plate in the camera had a pixel width and therefore a resolution of $5.19 \mu \mathrm{m}$.

For fluorescent microsphere detection, image resolution was a crucial factor in order to quantify the $15 \mu \mathrm{m}$ microspheres in the slide images. In piglet brain slide images, we achieved a resolution of $5.7 \mu \mathrm{m}$, sufficient for detecting the microspheres. Each microsphere spanned approximately 6-9 pixels across its diameter, depending on light spill-out to adjacent tissue, so microspheres appeared to have a diameter of $35 \mu \mathrm{m}-45 \mu \mathrm{m}$.

Table 2 shows image resolution results with extensions. Notice the larger FOV when using the slide scanner set-up (no. $1-5)$ when compared with a commercial bright-field microscope (no. 6-7).

By using microscope objective lenses for bright-field microscopy, the best achieved resolution was $0.7 \mu \mathrm{m}$, but the image quality was affected by the blue lighting from the UV light bulbs.

\subsection{Fluorescent microspheres}

Figure 2 shows a typical image of a slide from our study on newborn piglet brain. As seen in Figure 2, the microspheres are clearly depicted. The image quality was more than sufficient for an automatic detection algorithm to quantify microspheres in each slice and thereby estimate rCBF. Approximately 3,200 images were processed in MATLAB corresponding to 1,600 slides. Results of $\mathrm{rCBF}$ will be reported in a separate publication.

\subsection{Validation}

For validation of the microsphere detection algorithm, we compared results to manual counts of microspheres in a sample series of 60 brain slides from one piglet, which was reported in a congress abstract. ${ }^{[6]}$ In each slide, the amount of microspheres was counted manually using a conventional fluorescence microscope and secondly by the algorithm, resulting in two datasets. The mean difference (SD) between manual and algorithm counts was 0.32 (1.5) microspheres per slide in absolute numbers with a range from -5 to $5 \mathrm{mi}-$ crosphere per slide. The relative total count difference was $-3.1 \%$. See Table 3 for results of the validation study.

\subsection{Bright-field}

We tested the scanner for bright-field imaging by applying light diffuser film which produced a homogeneous blue visible light from the UV light bulbs (see Figure 3b). Figure 
4 shows a hematoxylin and eosin (HE) stained image of a human cerebellum compared with an image from a conventional microscope. The best trade-off between magnification and FOV was achieved by using the extension tubes, where we obtained a resolution of $2.9 \mu \mathrm{m}$ (see Table 1, set-up no. 1-5). A conventional bright-field microscope can achieve a maximum resolution of $0.2 \mu \mathrm{m}$, however, we achieved a resolution of $0.7 \mu \mathrm{m}$ with a $40 \times$ magnification objective (see Figure 5 of human epiglottis), although image quality was poorer compared with the conventional microscope and the image was bluish with a small FOV and blurred edges.

Table 2: Slide scanner and microscope technical specifications

\begin{tabular}{lllll}
\hline Set-up no. & Extensions $(\mathbf{m m})$ or Objective lens & Resolution $(\boldsymbol{\mu m})$ & Viewing width $(\mathbf{m m})$ & FOV $\left._{(\mathbf{m m}}\right)^{2}$ \\
\hline 1 & 13 & 9.0 & 38.4 & 993 \\
2 & 21 & 7.5 & 32.2 & 692 \\
3 & 34 & 5.7 & 24.5 & 400 \\
4 & 52 & 3.9 & 16.8 & 189 \\
5 & 65 & 2.9 & 12.3 & 101 \\
6 & Microscope 1.25× objective lens & 3.6 & 5.0 & 19.1 \\
7 & Microscope 5× objective lens & 0.9 & 1.1 & 1.3 \\
\hline
\end{tabular}

Note. The table illustrates the slide scanner equipped with a $55 \mathrm{~mm}$ camera lens and different extension set-ups (no.1-5), and listing the resulting resolution (pixel width in $\mu \mathrm{m}$ ), viewing width $(\mathrm{mm})$ and field-of-view $\left(\mathrm{FOV}, \mathrm{mm}^{2}\right.$ ). Set-up no. 6 and 7 compares the slide scanner with a conventional microscope. Notice set-up no. 4 which shows good resolution, larger viewing width, and FOV when compared with a conventional microscope in set-up no. 6.

Table 3: Validation study of MATLAB fluorescent microsphere detection algorithm

\begin{tabular}{llll}
\hline $\begin{array}{l}\text { Fluorescent } \\
\text { microspheres }\end{array}$ & $\begin{array}{l}\text { Manual } \\
\text { counts }\end{array}$ & $\begin{array}{l}\text { Algorithm } \\
\text { counts }\end{array}$ & $\begin{array}{l}\text { Difference } \\
\text { (\%) }\end{array}$ \\
\hline Carmine/Red & 860 & 823 & -4.3 \\
Yellow/Green & 320 & 305 & -4.7 \\
Blue & 274 & 281 & +2.6 \\
Total & 1,454 & 1,409 & -3.1 \\
\hline
\end{tabular}

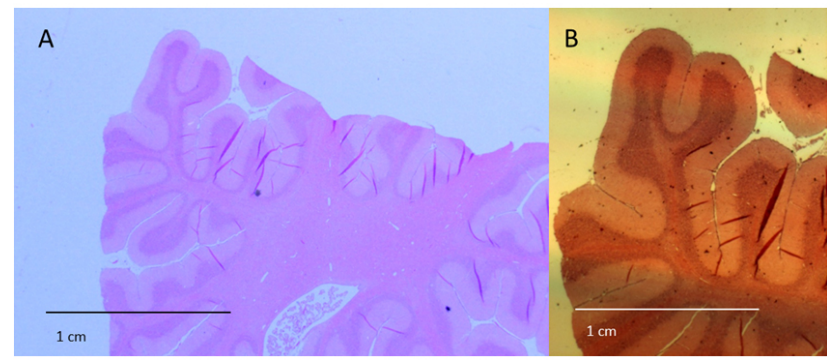

Figure 4: Hematoxylin and eosin staining of human cerebellum

The left panel (A) shows the imaging result when using a DSLR camera with $13 \mathrm{~mm}$ extension and a resolution of $9.0 \mu \mathrm{m}$. The field-of-view when using extensions gave a very good overview of a large tissue area with good resolution. For comparison, the right panel (B) shows a conventional microscope image of the same slide with $1.25 \times$ objective lens.

\section{Discussion}

We have developed a new low-cost, self-made slide scanner, which may be useful for researchers in need of digital imaging of tissue slides. Commercially available microscopes are costly and may not be readily available. The cost of the slide scanner presented here was approximately USD 1,000, which is a fraction of the cost of commercial slide scanners. By using a standard DLSR camera, we have obtained a good image resolution of $2.9 \mu \mathrm{m}$ and a large FOV of $101 \mathrm{~mm}^{2}$.

We have demonstrated slide scanner imaging of fluorescent microsphere in brain tissue and developed and tested algorithms dedicated to detection of the fluorescent microspheres. In a validation study, our results showed reasonable agreement with "gold standard" manual counting.

Fluorescent or dyed microspheres has been used to estimate tissue perfusion in a wide range of study types. ${ }^{[7-10]}$ The technique is a widely known and has been extensively used, but has mostly been applied with use of radioactive labeled microspheres however, fluorescent microspheres have been validated for the same purpose. ${ }^{[4]}$

For analysis, the common approach is to extract the microspheres from the tissue using digestion and counting the spheres in the desired tissue by flow cytometry. This is a well-established process and allows samples to be made from the organ of interest. A lower limit to the size of samples depends on the content of spheres. A minimum of 400 spheres is necessary to ensure statistical certainty. ${ }^{[11]}$ The digestion does however not preserve the anatomical information from i.e. white and gray matter tissue. To address this issue we developed the presented imaging method and algorithm to analyze brain tissue and image with a slide scanner as previously suggested. ${ }^{[1]}$ In this manner it was possible to visualize tissue anatomy and structure, which ideally would allow measurements of tissue perfusion in different tissue compartments.

However, the piglet animal model used in our studies did not allow estimation of perfusion in the gray and white mat- 
ter tissue compartments, because of the small thickness of the white matter $(2 \mathrm{~mm}-3 \mathrm{~mm})$ and the resulting few microsphere counts, which did not exceed the limit of 400 to ensure sufficient statistics. It was however possible to distinguish white and gray matter in the unstained tissue slides (see Figure 2). For piglets and other animal models it might be possible to appropriately stain tissue and subsequently develop algorithms to automatically count microspheres in different tissue compartments, when contrast between tissues are enhanced and larger areas be explored.

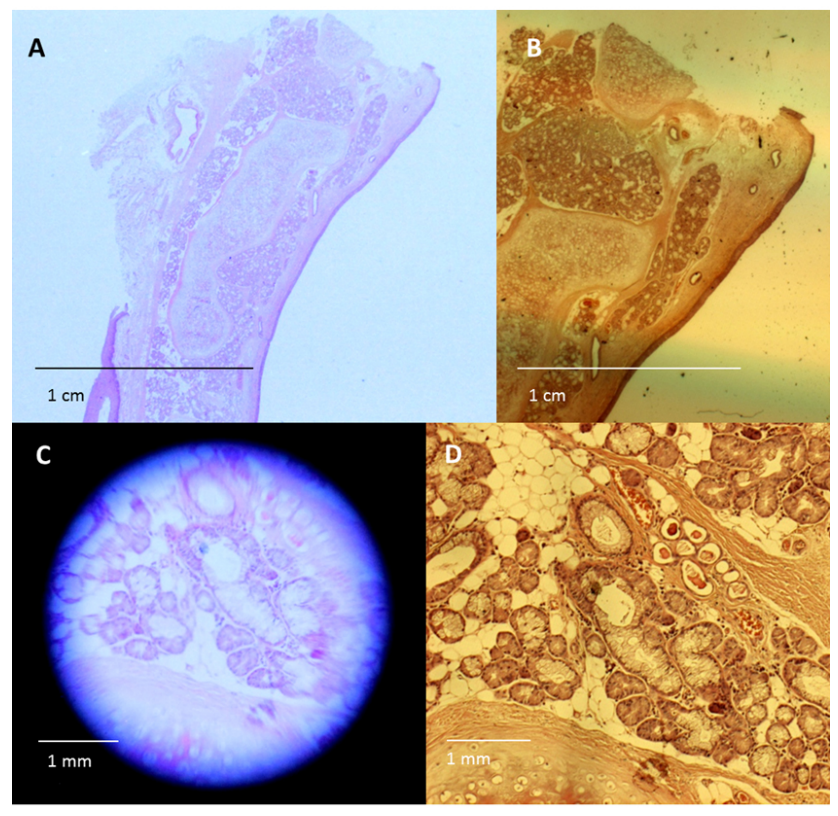

Figure 5: Human epiglottis in HE staining

The top panel, left image (A) shows the imaging result when using a DSLR camera with $13 \mathrm{~mm}$ extension and resolution of $9.0 \mu \mathrm{m}$. For comparison the right image $(B)$ shows a conventional microscope image of the same slice with $1.25 \times$ objective lens. The bottom panel left image $(C)$ shows the imaging result when using a DSLR camera with 40× microscope objective and resolution of $0.7 \mu \mathrm{m}$. With larger magnification individual cells were visualized though the image quality was poorer and had blurred edges when compared with conventional microscope image of the same slice using a $5 \times$ objective lens (bottom panel, right image, $D$ ).

Regarding the camera quality, the image resolution was limited by the applied camera sensor. However, a larger camera sensor would give a larger FOV at the same resolution, while higher resolution sensors, i.e. mega pixels (MP) per sensor size, would result in higher resolution. This could be of value in imaging of smaller animal models for example rats and mice, where smaller microspheres $(5 \mu \mathrm{m}-10 \mu \mathrm{m})$ are used for estimation of tissue perfusion.

The thickness of the tissue slices used in this study was $200 \mu \mathrm{m}$, which may have caused an underestimation of microsphere count when part of the microspheres where imbedded in deeper parts of the slice. Thus, this may have caused the algorithm to misclassify microspheres if there appeared blurred. However, we observed that after a few days from cutting and mounting the tissue slides, tissue appeared more transparent, which may have caused brighter and better delineated microspheres. We imaged the microspheres at least 5 days after cutting.

UV light bulbs and light diffuser film were tested for brightfield imaging with a resulting high resolution, however image quality was limited by the lower quality of the light used. With an optimized light source e.g. light emitting diodes, and tissue staining, the imaging quality could be improved, enabling better use in bright-field and histology imaging.

The slide scanner cannot compete with professional confocal or high magnification microscopes, but numerous applications of the slide scanner may be possible, for example fluorescence imaging in both plant biology and biological science using animal models.

\section{Conclusion}

We have presented a new self-made slide scanner and demonstrated its suitability for imaging piglet brain tissue slides and quantifying fluorescent microspheres. Computer algorithms to detect fluorescent microspheres in images of tissue slides were in reasonable concordance with manual counting. Limitations were seen in the capability of regional estimation of microspheres in different tissue compartments and in the resolution of the camera used and image quality obtained. The slide scanner can be regarded as a low-cost versatile alternative when digital slide imaging is needed, by providing a large FOV and reasonable image resolution when compared with high-end microscopes.

\section{Acknowledgements}

Dr. William Henning and Dr. Julie Bjerglund Andersen contributed equally to this work. The authors wish to thank the Department of Clinical Physiology, Nuclear Medicine and PET and Peter Johansen, MD, PhD, Department of Clinical Genetics, Rigshospitalet, University of Copenhagen and René Villadsen, PhD, Department of Cellular and Molecular Medicine, University of Copenhagen for providing the opportunity and support to develop the slide scanner. 


\section{References}

[1] Eucker SA, Hoffman BD, Natesh R, et al. Development of a fluorescent microsphere technique for rapid histological determination of cerebral blood flow. Brain Res. 2010 Apr 22; 1326: 128-34. PMid: 20193669. http://dx.doi.org/10.1016/j.brainres . 2010.02 .059

[2] Roukos V, Misteli T. Deep Imaging: the next frontier in microscopy. Histochem Cell Biol. 2014 Aug; 142(2): 125-31. PMid: 24989801. http://dx.doi.org/10.1007/s00418-014-1239-5

[3] Rudolph AM, Heymann MA. The circulation of the fetus in utero. Methods for studying distribution of blood flow, cardiac output and organ blood flow. Circ Res. 1967 Aug; 21(2): 163-84. PMid: 4952708. http://dx.doi.org/10.1161/01.RES.21.2.163

[4] Glenny RW, Bernard S, Brinkley M. Validation of fluorescentlabeled microspheres for measurement of regional organ perfusion. J Appl Physiol. 1993 May; 74(5): 2585-97. PMid: 8335595.

[5] Sharma G, Martin J. MATLAB®: A Language for Parallel Computing. International Journal of Parallel Programming. 2009 Sep 1; 37(1): 3-36. http://dx.doi.org/10.1007/s10766-008-0 082-5
[6] Henning WS, Andersen JB, Christensen AN, et al. Validation of a new technique to estimate regional cerebral blood flow in piglets using fluorescent microspheres. Journal of Nuclear Medicine. 2014; 55(supplement 1): 1808.

[7] Prinzen FW, Glenny RW. Developments in non-radioactive microsphere techniques for blood flow measurement. Cardiovascular research. 1994; 28(10): 1467-75. http://dx.doi.org/10.1093/c $\mathrm{vr} / 28 \cdot 10.1467$

[8] Prinzen FW, Bassingthwaighte JB. Blood flow distributions by microsphere deposition methods. Cardiovascular research. 2000; 45(1): 13-21. http://dx.doi.org/10.1016/S0008-6363(99 ) 00252-7

[9] Santulli G, Ciccarelli M, Palumbo G, et al. In vivo properties of the proangiogenic peptide QK. J Transl Med. 2009; 7: 41. PMid: 19505323. http://dx.doi.org/10.1186/1479-5876-7-41

[10] Santulli G, Cipolletta E, Sorriento D, et al. CaMK4 Gene Deletion Induces Hypertension. J Am Heart Assoc. 2012 Aug; 1(4): e001081. PMid: 23130158. http://dx.doi.org/10.1161/JAHA.112.00 1081

[11] Buckberg GD, Luck JC, Payne DB, et al. Some sources of error in measuring regional blood flow with radioactive microspheres. $\mathrm{J}$ Appl Physiol. 1971 Oct; 31(4): 598-604. PMid: 5111009. 